\title{
Global 8.4-GHz VLBI observations of JVAS B0218+357
}

\author{
A. D. Biggs, ${ }^{1,2 \star}$ O. Wucknitz,${ }^{3,1} \dagger$ R. W. Porcas, ${ }^{4}$ I. W. A. Browne,${ }^{1}$ N. J. Jackson, ${ }^{1}$ \\ S. Mao ${ }^{1}$ and P. N. Wilkinson ${ }^{1}$ \\ ${ }^{1}$ University of Manchester, Jodrell Bank Observatory, Macclesfield, Cheshire SK11 9DL \\ ${ }^{2}$ Joint Institute for VLBI in Europe, Postbus 2, 7990 AA Dwingeloo, The Netherlands \\ ${ }^{3}$ Hamburger Sternwarte, Universität Hamburg, Gojenbergsweg 112, D-21029 Hamburg, Germany \\ ${ }^{4}$ Max-Planck-Institut für Radioastronomie, Auf dem Hügel 69, D53121, Bonn, Germany
}

27 October 2018

\begin{abstract}
In this paper we present new observations of the gravitational lens system JVAS B0218+357 made with a global VLBI network at a frequency of $8.4 \mathrm{GHz}$. Our maps have an rms noise of $30 \mu \mathrm{Jy}$ beam $^{-1}$ and with these we have been able to image much of the extended structure of the radio jet in both the A and B images at high resolution $(\sim 1$ mas). The main use of these maps will be to enable us to further constrain the lens model for the purposes of $H_{0}$ determination. We are able to identify several sub-components common to both images with the expected parity reversal, including one which we identify as a counter-jet. We have not been successful in detecting either the core of the lensing galaxy or a third image. Using a model of the lensing galaxy we have back-projected both of the images to the source plane and find that they agree well. However, there are small, but significant, differences which we suggest may arise from multi-path scattering in the ISM of the lensing galaxy. We also find an exponent of the radial mass distribution of $\beta \approx 1.04$, in agreement with lens modelling of published 15-GHz VLBI data. Polarisation maps of each image are presented which show that the distributions of polarisation across images A and B are different. We suggest that this results from Faraday rotation and associated depolarisation in the lensing galaxy.
\end{abstract}

Key words: quasars: individual: JVAS B0218+357 - gravitational lensing - galaxies: ISM

\section{INTRODUCTION}

Few gravitational lens systems are as well studied as JVAS B0218+357 (Patnaik et al. 1993, perhaps the best example of a lens system for which the method of Refsdal (1964) can be used to determine the Hubble parameter, $H_{0}$. Unlike many other methods, this is done in a single step and involves well understood, and relatively simple, astrophysics. We point out that there remain significant uncertainties in $H_{0}$ as determined by traditional methods (e.g. Shanks et al. 2001). For example, the use of Cepheid-calibrated distances to determine the distance to galaxies hosting Type Ia supernovae has resulted in final values of $H_{0}$ that do not agree at the $1 \sigma(\sim 10$ per cent) level (Parodi et al, 2000; Freedman et al.2001). This is despite a large overlap between the samples of SNIa.

Whilst the gravitational lens route to $H_{0}$ can be a particularly "clean" one, and despite many lens systems now having measured time delays, almost all suffer in one way or another from effects that significantly increase the uncertainty in the final determination of

\footnotetext{
* E-mail: biggs@jive.nl

$\dagger$ Present address: Universität Potsdam, Institut für Physik, Am Neuen Palais 10, 14469 Potsdam, Germany.
}

$H_{0}$. In most cases the main source of uncertainty is in the modelling of the gravitational potential responsible for the image splitting and distortion. A lack of observational data to constrain the lens model and multiple-galaxy lenses are both significant factors in this regard (see e.g. Schechter 2000). A list of the time delays measured to date can be found in Courbin, Saha \& Schechter 2002.

With JVAS B0218+357 on the other hand, the potential exists to reduce the uncertainty in the lens model to a level comparable to that in the time delay; this currently stands at 3 per cent ( $\tau=10.5 \pm 0.4 \mathrm{~d}$; Biggs et al., 1999). Other authors find a value for the time delay consistent with this Cohen et al. 2000. This reduction in model uncertainty is possible due to the large number of observational model constraints available from multi-frequency, multi-resolution imaging. Both images (A and $\mathrm{B}$ ) of the $z=0.96$ background quasar/BL Lac are easily resolved with VLBI into two subcomponents (e.g. Patnaik, Porcas \& Browne 1995; Kemball, Patnaik \& Porcas 2001) which constrain the radial mass profile to be close to isothermal. The lens systems with the largest numbers of model constraints are those that contain Einstein rings, such as B0218+357. The resolved arcsec-scale structure of the ring probes the lens potential along multiple lines of sight and along all azimuthal position angles relative to the lens centre. The ring (see 
Fig. (4) has been mapped at high resolution using combined MERLIN and VLA data at $5 \mathrm{GHz}$ (Biggs et al. 2001) and the model constrained using a version of the LensClean algorithm (Kochanek \& Narayan 1992); preliminary results can be found in Wucknitz 2001). Finally, the deflecting mass is concentrated in a single, isolated galaxy and so the effect of tidal shear due to nearby structure is very small ( $\sim 1$ per cent, Lehár et al. 2000 ). This results in a relatively uncomplicated mass model compared to other lens systems. A VLA map of B0218+357 can be seen in Fig. 7 .

The main stumbling block to date in measuring $H_{0}$ with JVAS B0218+357 has been that, due predominantly to the small size of the system (A-B separation of 334 mas), it has been difficult to measure the position of the $z=0.6847$ lensing galaxy relative to the lensed images accurately using optical $(H S T)$ data. For example, the positions derived from two NICMOS observations differ by 46 mas Lehár et al. 2000) and this uncertainty translates into a large uncertainty in $H_{0}$. With LensClean we have estimated the galaxy position to a theoretical accuracy of a few mas, but this relies to some extent on the lens model and so could be biased.

In this paper we present new 8.4-GHz observations of JVAS B0218+357 taken with a global VLBI array that were designed to increase the available model constraints by other means. With the great sensitivity of a global array we hoped we might detect:

(i) extended structure associated with the radio jet in both A and B. This is detected in $5-\mathrm{GHz}$ data from the First Caltech-Jodrell Bank (CJ1) VLBI survey Xu et al. 1995) that we have re-analysed to produce maps (Fig. 1) that are superior to those published in $\mathrm{Xu}$ et al.. With a resolution of $\sim 5$ mas these new maps reveal a jet that extends out to 30 mas from the core in each image.

(ii) a third image, as detected in, e.g. APM 08279+5255 (Ibata et al. 1999;; Lewis et al.(2002) and MG 1131+0456 (Chen \& Hewitt 1993; Chen, Kochanek \& Hewitt 1995, and

(iii) the galaxy core. Emission from the lensing galaxy has been detected in several systems e.g. B0957+561 (Harvanek et al. 1997) and CLASS B2045+265 (Fassnacht et al, 1999). A third radio component is detected in PMN J1632-0033, but it is not known if this is a lensed image or the lens galaxy (Winn et al. 2002).

The observations have also been used to investigate the masscale polarisation structure of this system. Whilst the cores of most quasars/BL Lacs are polarised at a level of about 2-3 per cent Saikia \& Salter 1988, images A and B of B0218+357 are both much more highly polarised, reaching $\sim 10$ per cent at frequencies $8.4 \mathrm{GHz}$ and above (Patnaik et al. 1993, Biggs et al. 1999). Also, the polarisation position angles of the images are not the same, in conflict with the expected behaviour of a lens system which preserves the polarisation position angle on the sky (Dyer \& Shaver 1992). It is thought that Faraday rotation in the magnetoionic medium of the lensing galaxy itself rotates the position angles of the images by different amounts (Patnaik et al. 1993. Thus the extended nature of the emission in images $\mathrm{A}$ and $\mathrm{B}$ allows the interstellar medium (ISM) of a high-redshift galaxy to be studied on parsec scales. Both images also depolarise at low frequencies.

\section{OBSERVATIONS AND DATA REDUCTION}

The goals of this experiment required a combination of high sensitivity, high resolution and excellent $(u, v)$ coverage in order to reliably image complex and faint structures. For this reason we used a global VLBI array including the ten antennas of the VLBA, six from the European VLBI Network (EVN), the VLA (Y) in phased-
Table 1. Global VLBI array antennas and performance at $8.4 \mathrm{GHz}$. Observations were conducted in dual circular polarisation at all antennas apart from $\mathrm{Nt}$, $\mathrm{On}$ and $\mathrm{Yb}$ where only right circular polarisation was available.

\begin{tabular}{|c|c|c|}
\hline Antenna & Diameter $(\mathrm{m})$ & SEFDâ (Jy) \\
\hline VLBA & 25 & 307 \\
\hline $\operatorname{VLA}\left(\mathrm{Y}_{9}\right)$ & $75)^{c}$ & 41 \\
\hline Effelsberg (Eb) & 100 & 20 \\
\hline Medicina (Mc) & 32 & 270 \\
\hline Noto $(\mathrm{Nt})^{\sqrt{d}}$ & 32 & 770 \\
\hline Onsala $(\mathrm{On})^{d}$ & 20 & 1630 \\
\hline Westerbork (Wb) & $94^{c}$ & 120 \\
\hline Yebes $(\mathrm{Yb})^{d}$ & 14 & 3300 \\
\hline Robledo (Ro) & 70 & 23 \\
\hline Goldstone (Go) & 70 & 20 \\
\hline
\end{tabular}

\footnotetext{
${ }^{a}$ System equivalent flux density

$b$ Inner nine antennas of VLA phased together

$c$ Diameter corresponding to summed geometric area of telescopes

$d$ Only right circular polarisation was available
}

array mode and the two 70-m antennas of the Deep Space Network (DSN) at Goldstone and Robledo. Details of these antennas are given in Table 1 with their diameters and system equivalent flux densities (SEFD). We chose to observe at $8.4 \mathrm{GHz}$ instead of at $5 \mathrm{GHz}$ (where the surface brightness sensitivity is better) due to the improved resolution and because there is less chance that the lensed emission would be affected by scattering; there is evidence that the image sizes in this system may be enlarged by scattering at low frequencies (Biggs et al., in preparation, although see Porcas \& Patnaik (1996b) for a 1.7-GHz map that illustrates the large size increase). Scattering would possibly reduce the surface brightness of the third image or the galaxy core which is clearly undesirable when searching for such weak features.

Due to the fact that the VLA was in its largest configuration ('A') at the time of the observations, using the full complement of 27 antennas as a single phased array was not possible as the synthesised beam would have been significantly smaller than the separation between the two images $\mathrm{A}$ and $\mathrm{B}$. Therefore we were forced to use only the inner nine antennas of the array, $\mathrm{Y}_{9}$, to give a large enough beam. It should be noted that in order to calibrate the absolute polarisation position angle, the strategy for which is described later in this section, three short additional observations of $3 \mathrm{C} 48$ and $3 \mathrm{C} 138$ were added to the inner subarray schedule. These two sources were not observed by any other antenna in the global array. The remaining 18 antennas of the VLA observed as an outer subarray operating in normal interferometric mode.

The observations took place during 11 November 2000 to 12 November 2000 over the time range 20:00-07:00 UT for the European antennas and 21:00-09:00 UT for those in the US. Both senses of polarisation were recorded with the exception of three of the stations where only right circular polarisation (RCP) was available ( $\mathrm{Nt}$, On and $\mathrm{Yb}$ ). Data were recorded in four $8 \mathrm{MHz}$ sub-bands with one-bit sampling giving a data rate of $128 \mathrm{Mbps}$. Of the 19 antennas scheduled (VLBA+EVN+DSN+Y), only 17 took part in the observations; Yebes suffered from a broken data formatter whilst the receiver at VLBA Owens Valley was non-operational. The various calibration sources observed are shown in Table 2 along with their flux densities. The data were correlated at the VLBA correlator in Socorro producing 16 0.5-MHz channels per sub-band (IF) 

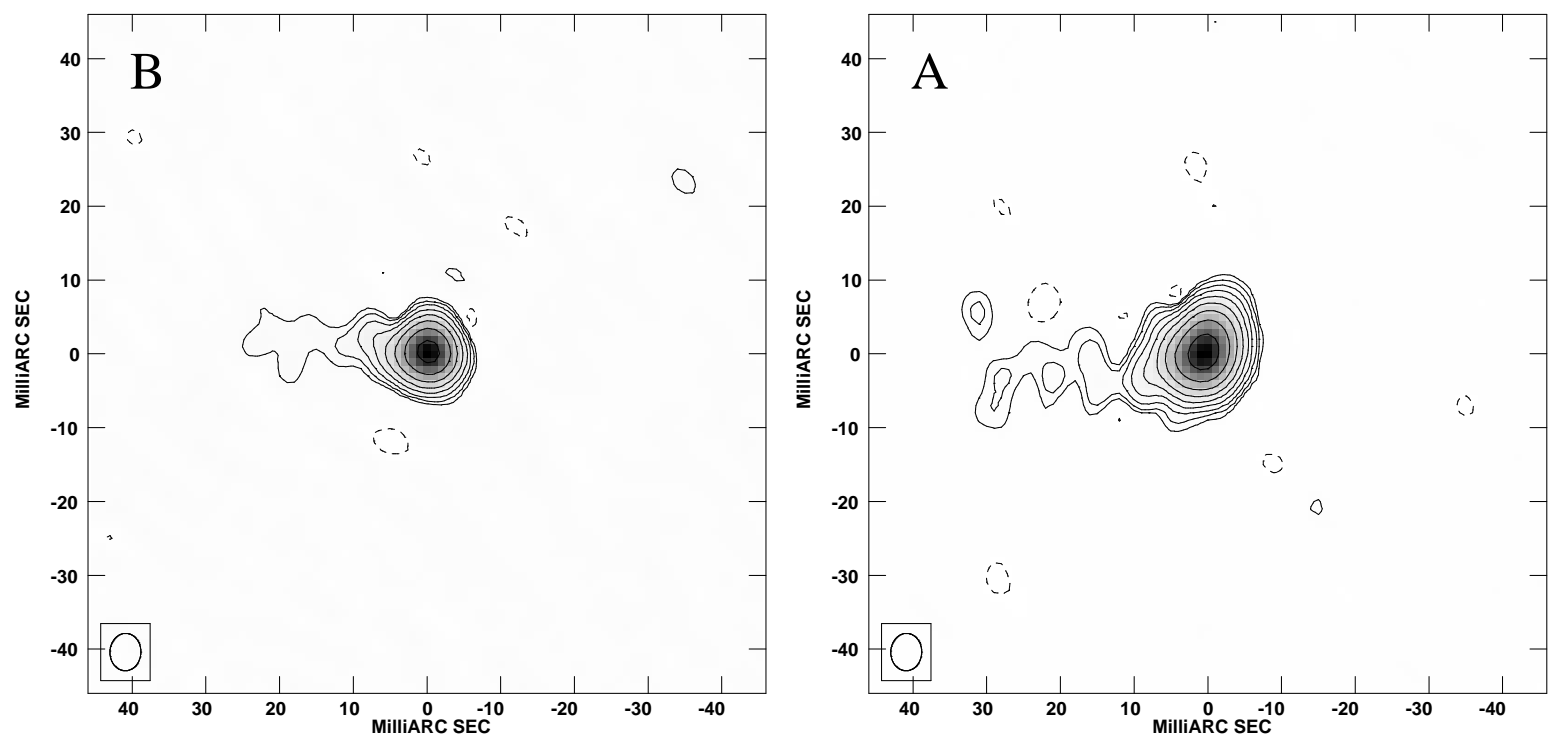

Figure 1. CJ1 VLBI maps of JVAS B0218+357 at $5 \mathrm{GHz}$. Left: B, right: A. The restoring beam is shown in the bottom-left corner of each map and has a FWHM of $5 \times 4.2$ mas at a position angle of -1 .9. Contours are plotted at multiples $(-1,1,2,4,8,16$, etc) of $3 \sigma$ where $\sigma$ is the off-source rms noise in the map $\left(330 \mu \mathrm{Jy}_{\text {beam }}{ }^{-1}\right)$. Both maps are plotted on the same angular scale.

Table 2. Approximate flux densities, hour angle coverage and role of the various calibration sources.

\begin{tabular}{llll}
\hline Source & Flux density (Jy) & \# Hour angles & Calibration role \\
\hline B0234+285 & 3.0 & 29 & amplitude \\
$3 \mathrm{C} 84$ & 19.0 & 8 & bandpass/L-R delay/D-term \\
$0059+581$ & 1.5 & 3 & polarisation position angle \\
$0300+470$ & 1.3 & 3 & polarisation position angle \\
$3 \mathrm{C} 48 \mathrm{q}^{a}$ & 3.2 & 2 & absolute polarisation position angle \\
$3 \mathrm{C} 138^{a}$ & 2.4 & 1 & absolute polarisation position angle
\end{tabular}

$a$ VLA inner antennas only

and using an averaging time of two seconds. All polarisation pairs (RR, LL, RL and LR) were produced.

Data reduction was performed using the NRAO AIPS package. Amplitude calibration was carried out using system temperatures $\left(T_{\mathrm{sys}}\right)$ recorded during the observations scaled by a priori measurements of the antenna gains. For the DSN antennas no $T_{\mathrm{sys}}$ measurements were available, but values were estimated from measurements of the total power. Delay residuals were calculated using short segments of B0234+285 data and applied to all sources. This source was then fringe-fitted, solving for delays, rates and phases for the entire dataset. Following this the calibrated B0234+285 data were mapped in IMAGR and self-calibrated in CALIB. The residual amplitude voltage gain solutions found during the self-calibration were large for many antennas (up to a factor of two for VLBA Pie Town) and so were subsequently applied to all sources in order to correct the initial amplitude calibration. Next the B0218+357 data were fringe-fitted, initially with a single component located at the location of the brighter A component relative to the correlation position, but later with a more realistic model made from mapped and self-calibrated data. The delay difference between the left and right polarisations was calibrated using a single scan of 3C84 data and two baselines to the reference antenna (VLBA North Liberty). The
3C84 data were also used to calibrate the bandpasses of the antennas.

Next the various steps to calibrate the polarisation were undertaken. Firstly, the leakage (or $D$-) terms were solved for using the 3C84 data which had first been mapped and self-calibrated; 3C84 was assumed to be unpolarised. Secondly the absolute position angle of the polarisation was calibrated using the data from the inner subarray of the VLA $\left(\mathrm{Y}_{9}\right)$ in normal interferometric mode ${ }^{\text {円. As }}$ mentioned earlier, the bright calibration sources $3 \mathrm{C} 48$ and $3 \mathrm{C} 138$ were included in the $\mathrm{Y}_{9}$ schedule and with these it was possible to determine the true position angle of the source $0059+581$. Once this was known it was possible to calibrate the position angle of the global array data by rotating the RCP-LCP phase difference until the $0059+581$ polarisation position angle agreed with that found with the VLA data.

At this point the calibrated $\mathrm{B} 0218+357$ data were averaged in frequency to produce one channel per IF and averaged in time over $10 \mathrm{~s}$ intervals. The regions around images A and B were CLEANed using the IMAGR wide-field mapping task and the

\footnotetext{
1 The antennas of the phased array also produce the usual baseline combinations as a matter of course.
} 
data self-calibrated in CALIB. Many cycles of mapping and selfcalibration were undertaken, initially only allowing the phases to vary, but later the amplitudes as well. It was found that the sensitivity of the Westerbork (Wb) telescope was changing by up to a factor of four, cycling over 20 min timescales. Due to this, and the fact that the polarisation leakage terms were excessively large (up to 45 per cent), the $\mathrm{Wb}$ data were flagged and took no further part in the analysis. Much of the previous calibration was then repeated to make sure that the $\mathrm{Wb}$ data was not biasing the calibration for the other antennas. Data from the Noto and Onsala antennas could not be included when making the Stokes $Q$ and $U$ maps as neither was able to observe both right and left circular polarisations.

Unlike the antennas that formed the global VLBI array $\left(\mathrm{VLBA}+\mathrm{EVN}+\mathrm{DSN}+\mathrm{Y}_{9}\right)$ the outer subarray of the VLA observed with a separate schedule, nodding between B0218+357 (15 mins on source) and $0234+285$ ( 2 mins on source) over a period of six hours. The data were calibrated in AIPS using $0234+285$ as the phase, amplitude and polarisation calibrator. The $D$-terms were solved for assuming a point-source for $0234+285$ (a good approximation to the polarised intensity image) whilst simultaneously solving for the source polarisation. The position angle of polarisation was again aligned using the inner VLA subarray $\left(\mathrm{Y}_{9}\right)$ data. These data were then mapped and self-calibrated, again in AIPS.

\section{RESULTS}

\subsection{Global VLBI maps (total intensity)}

The final VLBI maps are shown in Fig. 2 and are made from uniformly-weighted data in all cases. This results in an off-source rms noise of $30 \mu \mathrm{Jy}$ beam $^{-1}$ (compared to a theoretical noise of $\sim 10 \mu \mathrm{Jy}_{\text {beam }^{-1}}$ ) and a synthesised beam of $1.36 \times 0.41$ mas for the total intensity maps; these are shown in the top two panels. Here the images are dominated by two bright sub-components, with a separation of $\gtrsim 1$ mas in both $\mathrm{A}$ and $\mathrm{B}$. These were designated (A or B) 1 and 2 by Patnaik et al. (1995) and observations at higher frequencies $(15,22$ and $43 \mathrm{GHz})$ show the same two sub-component structure (Porcas \& Patnaik 1996a). The core is believed to be the most westerly sub-component (1) in each case, based on its compactness relative to sub-component 2 . Its spectrum also turns over at a higher frequency, as expected for a more compact component. As well as the two bright sub-components though, we have also detected large quantities of extended emission, in both $\mathrm{A}$ and $\mathrm{B}$. This, however, looks quite different in each of the images, image A being considerably more resolved than image B. It is exactly these differences that can be used as constraints on the lens model as they must be explicable, in the absence of effects such as scattering, by the action of the lensing galaxy.

Interpreting image B is straightforward as it looks like a typical core-jet radio source, with a knotty jet extending eastwards from the bright core region. This is in line with what we would expect given the new CJ maps of Fig. 1. However, in order to aid the eye in comparing images $\mathrm{A}$ and $\mathrm{B}$, the structures that we will now describe have been labelled on the maps in Fig. 22. The jet in B appears to bend, pointing in an approximate north-easterly direction immediately after emerging from the core (Jet 1), before gently curving over the next 5 mas to point in a more south-easterly direction (Jet 2). Finally the jet changes direction again, returning to a northeasterly trajectory before fading completely (Jet 3). Returning to the core region, there is a small counter-component that appears to emerge from the south-west of B1.
In image A sub-components 1 and 2 are, as already stated, clearly detected. However, they appear to sit in a large structure that is edge-brightened on its western side and extended to the north and south. This has been observed before at this frequency (Kemball et al. 2001) as well as at $15 \mathrm{GHz}$ (Patnaik et al.|1995), but the greater sensitivity of our maps makes the effect much more pronounced. The majority of this structure, as has been noted by previous authors, is in fact emission from the core region that has been tangentially stretched along a position angle $\sim-30^{\circ}$ (perpendicular to the A-lens galaxy separation) as part of the lensing process. Lens models predict just such a stretching of the brighter image in two-image lens systems, but it is not usually as resolved as that seen in $\mathrm{B} 0218+357$. We can quantify the magnitude of the stretching by noticing that the separations A1-A2 and B1-B2 are approximately equal ( $1.45 \pm 0.02$ mas). From observations with MERLIN and the VLA the flux density ratio between A and B at centimetre wavelengths is known to lie between three and four Patnaik et al. 1993. Biggs et al. 1999; Cohen et al. 2000). As this flux density ratio is due to an increase in the size of image A relative to $B$ then image A must be stretched by a factor 3-4 relative to $B$ in the observed direction. A similar conclusion was reached by Patnaik et al, (1995).

The other features identified in image $\mathrm{B}$ are also clearly visible in image $\mathrm{A}$ and with properties consistent with gravitational lensing. The jet emerges from the core region in A and is parity reversed as expected - whilst Jet 1 in B initially lies above a line joining $\mathrm{B} 1$ and $\mathrm{B} 2$, in $\mathrm{A}$ it lies below a line joining $\mathrm{A} 1$ and $\mathrm{A} 2$. The tangential stretching displaces this jet feature away from the 1-2 separation vector compared to $\mathrm{B}$. Proceeding eastwards the jet emission remains tangentially stretched (the Jet 2 region is seen in the CJ1 map (Fig. 1) as a bright extension to the east of the core) before fading as in B into discrete blobs. Note, however, the opposite direction of the very faint emission at the far end of the jet (Jet 3). In A this lies along a south-east line compared to north-east in $\mathrm{B}$. The counter-component in A is also visible, but is located to the north of the A1-A2 separation, as opposed to south of the B1-B2 separation vector.

In order to search for emission from either the core of the lens galaxy or a third image, we have also made maps of the region in between images $\mathrm{A}$ and $\mathrm{B}$. No evidence is found for either. If we assume that these components would have been detected if their peak surface brightness was greater than the 6- $\sigma$ rms noise level of $180 \mu \mathrm{Jy}$ beam $^{-1}$, we conclude that the third image has a flux density less than $180 \mu \mathrm{Jy}, 0.02$ per cent of the component A total flux density. Here we have assumed that the third image will be unresolved as its size should be that of $\mathrm{A}(\sim 10 \times 10$ mas $)$ divided by the relative de-magnification factor of $\sim 5000$ i.e. less than the size of the beam. Multi-path scattering (which we show is probably affecting the size of image A in Section 4.2) could though broaden the third image and reduce its surface brightness. If this is the case then the upper limit given above for the flux density of the third image is an under-estimate.

\subsection{Global VLBI maps (polarised intensity)}

Our polarised intensity maps $\left(P=\sqrt{Q^{2}+U^{2}}\right.$, bottom row of Fig. 2) show no polarised jet emission outside of the core region. Sub-components 1 and 2 though are prominent and in image $A$ are, as in the total intensity maps, tangentially stretched. In image B polarised inter-component emission between $\mathrm{B} 1$ and $\mathrm{B} 2$ is visible and appears as a small jet-like extension from sub-component B1, again supporting the hypothesis that this is the core. 

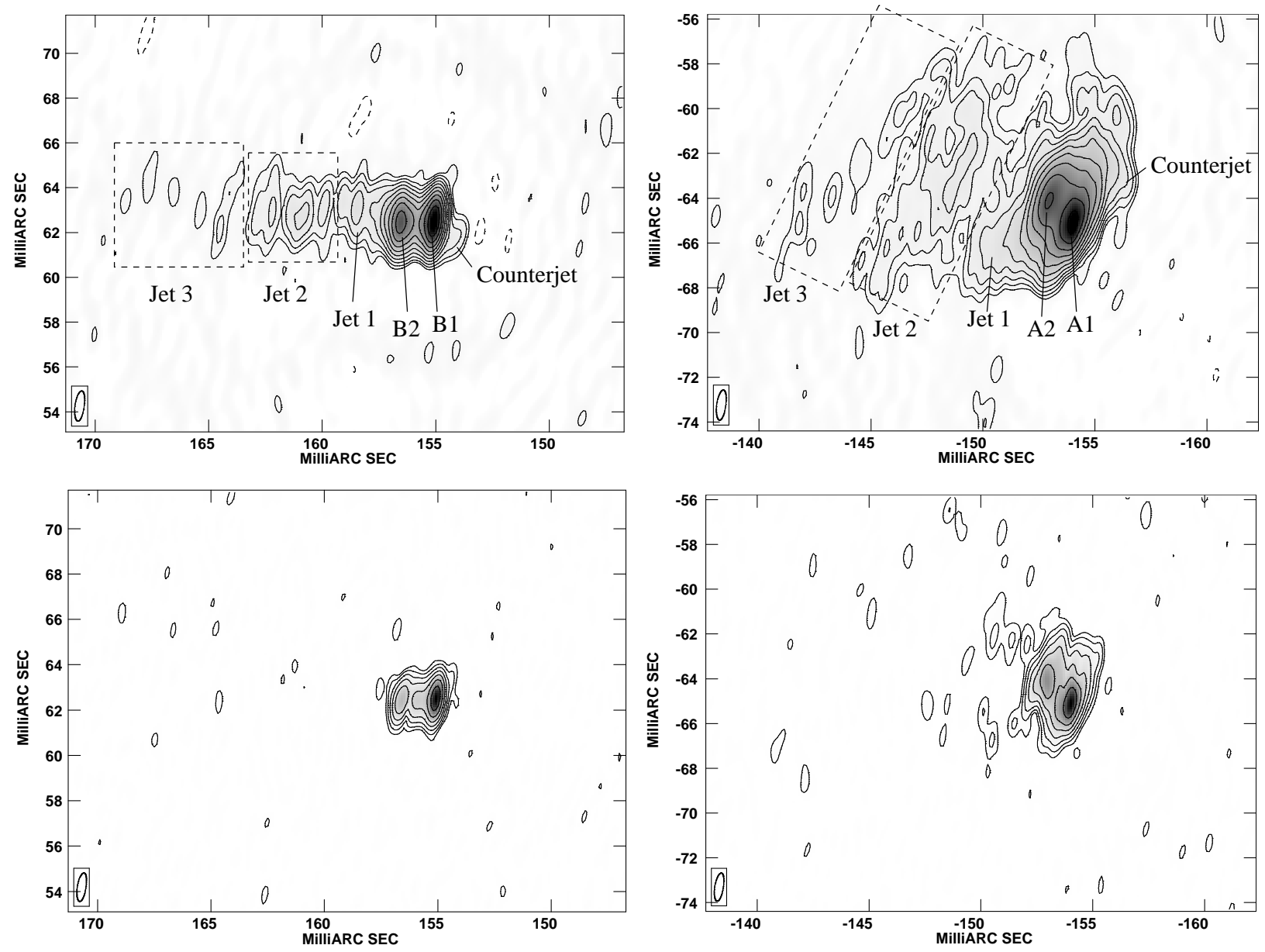

Figure 2. Global VLBI maps of JVAS B0218+357 at $8.4 \mathrm{GHz}$. Left: B, right: A. Top: Total intensity (Stokes $I)$. Contours are plotted at multiples ( -1 , 1, 2 , $4,8,16$, etc) of $3 \sigma$ where $\sigma$ is the off-source rms noise in the map $\left(30 \mu \mathrm{Jy} \mathrm{beam}^{-1}\right)$. The restoring beam is shown in the bottom-left corner and has a FWHM of $1.36 \times 0.41$ mas at a position angle of -7.5 . Bottom: Polarised intensity plotted on the same angular scale as the figures in the top row. Contours are also plotted at the same levels as in the top figures, but the restoring beam here has a FWHM of $1.27 \times 0.38$ mas at a position angle of -8.9 .

The top row of Fig. 3 shows polarisation E-field vectors overlaid on total intensity contours. The effect of the differential Faraday rotation between the images can be seen as a rotation of the polarisation vectors in image $\mathrm{A}$ by $\sim 50^{\circ}$ clockwise relative to image B. In image B it is possible to identify changes in the polarisation position angle along the jet axis, including across B2, the least compact sub-component. Generally though the position angle tends to lie approximately parallel to the jet axis. This "alignment" is entirely coincidental as both the jet axis and polarisation position angles are changed from their true values, the first by the gravitational effect of the lens and the second by Faraday rotation. In image A the distribution of the position angle is more complicated, there being an anomalous region of polarisation that stretches northwards from the midway point between A1 and A2. Here the polarisation vectors are oriented at $\sim 0^{\circ}$ as opposed to elsewhere in this image where the majority of the polarisation is oriented between position angles of $\sim 30-40^{\circ}$. This anomalous region is also seen in the maps of Kemball et al, 2001).

The bottom row of Fig. 3 shows (in greyscale) percentage polarisation maps of $\mathrm{A}$ and $\mathrm{B}$, plotted on the same angular scale as the polarisation position angle figures above. The inner jet of $\mathrm{B}$ is particularly prominent here as it is very highly polarised, with a peak of 20 per cent. Interestingly, the distribution of percentage polari- sation looks very different in image A where the peak polarisation is lower at only 12 per cent. There is also no sign of a significantly more highly polarised sub-component between A1 and A2.

\subsection{Global VLBI maps (model fitting)}

We measured the flux densities and sizes for sub-components 1 and 2 by fitting elliptical Gaussians to the total intensity maps using the AIPS tasks JMFIT and IMFIT. We choose to fit to the maps, rather than to the $(u, v)$ data, as the biasing effect of the extended jet emission can then be more easily reduced. This is achieved by removing all pixels from the images whose surface brightness is less than $2.88 \mathrm{mJy}^{\text {beam }}{ }^{-1}$. This corresponds to the sixth-lowest positive contour in the total intensity maps of Fig. 2 above which there is little evidence of jet emission in either image A or B. The possibility of contamination from the inner jet remains and the effect of this, although probably small due to this being relatively weak, will be different in each image. The positions of the Gaussians are constrained to lie at the maxima of sub-components 1 and 2 .

The measured flux densities and deconvolved sub-component sizes are shown in Table 3. Errors have been estimated by comparing the results from IMFIT and JMFIT, but are probably underesti- 

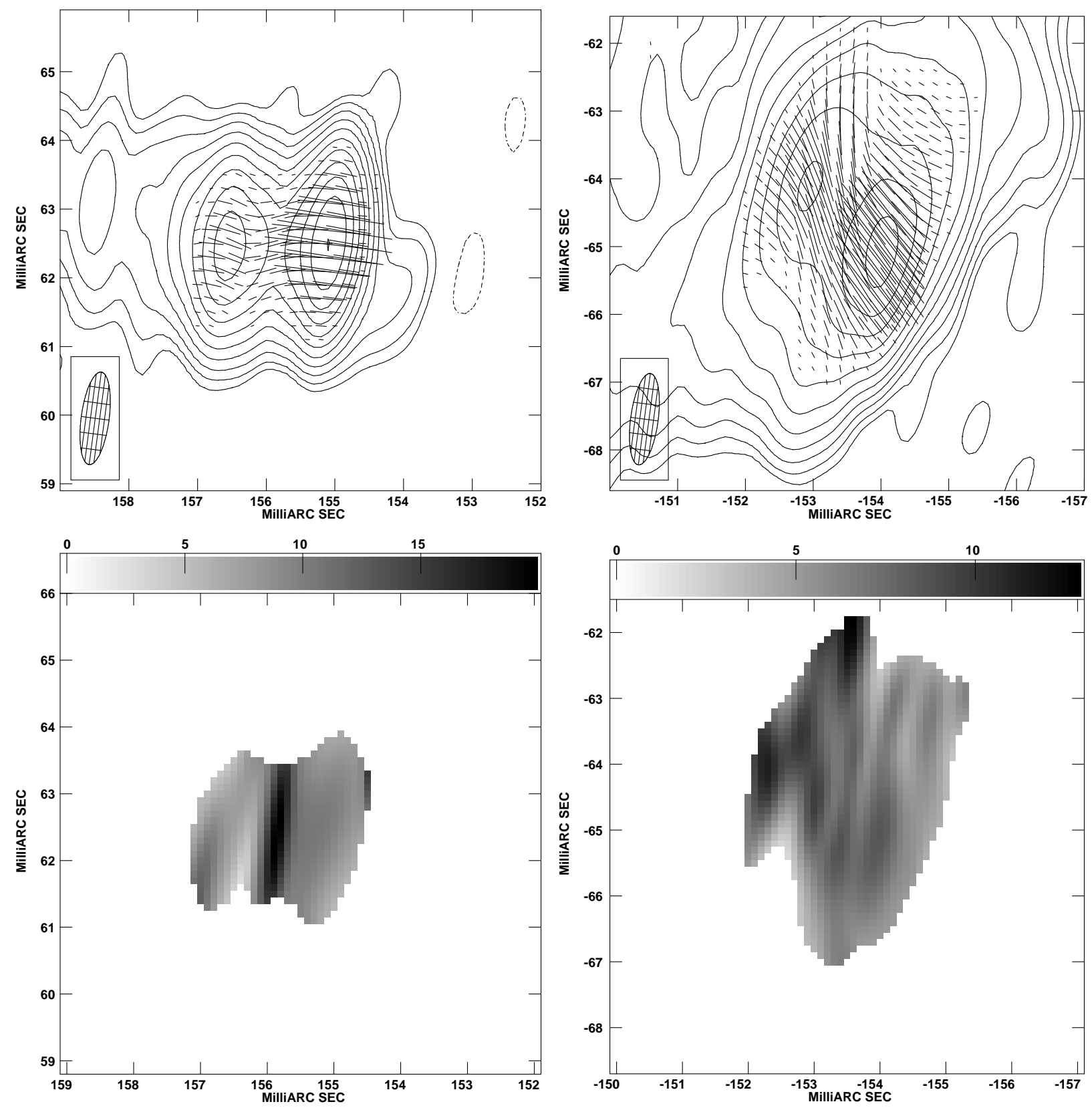

Figure 3. Global VLBI maps of JVAS B0218+357 at $8.4 \mathrm{GHz}$. Left: B, right: A. Top: Close up of the total intensity maps (contours) of Fig. 2 with polarised intensity E-vectors overlaid. A vector of length 1 mas equals 5 mJy beam ${ }^{-1}$. Bottom: Percentage polarisation greyscales plotted on the same angular scale as the figures in the top row. For each image the darkest greyscale corresponds to the peak intensity in the image.

Table 3. Results of elliptical Gaussian fitting to the VLBI maps. Tabulated are flux densities $\left(\mathrm{S}_{\nu}\right)$, deconvolved major $(a)$ and minor $(b)$ axes and orientation of ellipticity $(\phi)$.

\begin{tabular}{llllr}
\hline Image & $\mathrm{S}_{\nu}$ (mJy) & $a$ (mas) & $b$ (mas) & $\phi\left(^{\circ}\right)$ \\
\hline $\mathrm{A} 1$ & $270 \pm 5$ & $1.3 \pm 0.1$ & $0.5 \pm 0.1$ & $-33 \pm 2$ \\
$\mathrm{~A} 2$ & $280 \pm 5$ & $2.3 \pm 0.1$ & $1.2 \pm 0.1$ & $-30 \pm 2$ \\
\hline $\mathrm{B} 1$ & $114 \pm 3$ & $0.30 \pm 0.02$ & $0.16 \pm 0.02$ & $83 \pm 2$ \\
$\mathrm{~B} 2$ & $68 \pm 3$ & $0.80 \pm 0.02$ & $0.40 \pm 0.02$ & $81 \pm 2$
\end{tabular}

mates of the true uncertainties due to the systematic effects alluded to in the previous paragraph. If we consider only image $B$, where the errors are smaller, we see that sub-component 1 is the more compact and that its flux density is significantly lower than that measured by Kemball et al. (2001). As that of B2 has remained unchanged over the intervening five years, these maps support the identification of sub-component 1 as the core and the origin of the variability seen in the VLA monitoring campaigns.

\subsection{VLA maps (outer subarray)}

The total intensity map of the VLA-only data is shown in Fig. A with polarisation E-field vectors overlaid. It shows all the major features of B0218+357 on arcsec scales - the radio cores A and B, 


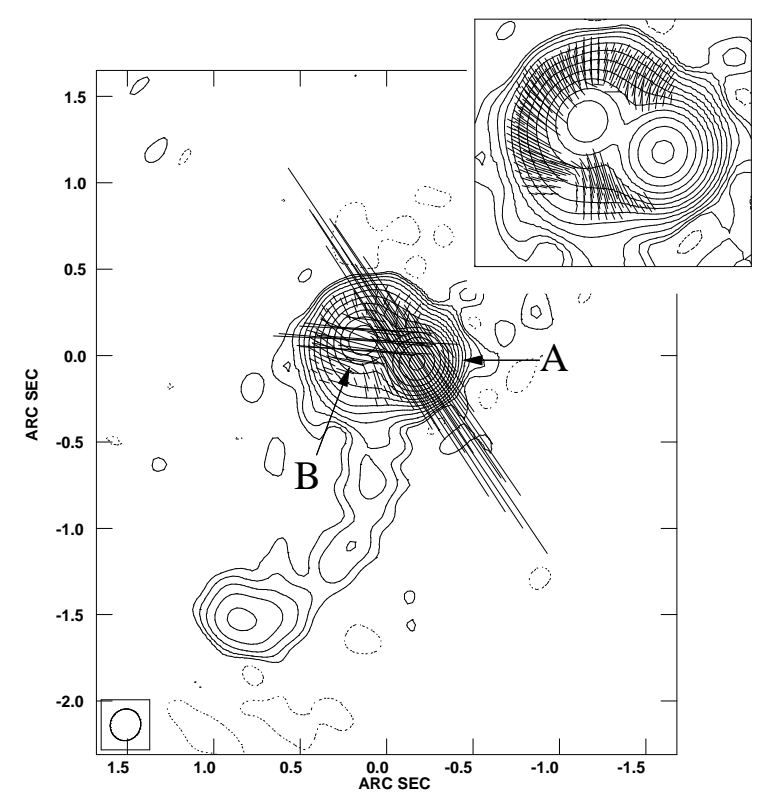

Figure 4. VLA (outer subarray) contour map of JVAS B0218+357 at $8.4 \mathrm{GHz}$ with polarisation E-vectors overlaid. The restoring beam is shown in the bottom-left corner of the map and has a FWHM of $186 \times 174$ mas at a position angle of $-26^{\circ} 2$. Contours are plotted at multiples $(-1,1$, $2,4,8,16$, etc) of $3 \sigma$ where $\sigma$ is the off-source rms noise in the map $\left(50 \mu \mathrm{Jy}_{\text {beam }}{ }^{-1}\right)$. The inset at the top right shows the region of the Einstein ring without the $\mathrm{A}$ and $\mathrm{B}$ polarisation vectors.

Table 4. Total flux density $(I)$, polarised intensity, $(P)$, percentage polarisation $(m)$ and polarisation position angle $(\phi)$ for images A and B as measured from the VLBI and VLA datasets. The $P$ and $\phi$ VLBI values are derived from measurements of the integrated $Q$ and $U$ flux densities. The VLA values have been calculated by model-fitting delta components to the $(u, v)$ data.

\begin{tabular}{lllll}
\hline Image & $I(\mathrm{mJy})$ & $P(\mathrm{mJy})$ & $m(\%)$ & $\phi\left(^{\circ}\right)$ \\
\hline A (VLBI) & 602 & 35.8 & 5.9 & 29 \\
A (VLA) & 744 & 34.1 & 4.6 & 33 \\
B (VLBI) & 191 & 17.1 & 9.0 & 83 \\
B (VLA) & 253 & 16.8 & 6.6 & 82
\end{tabular}

the Einstein ring and the radio jet. In addition, the region around the Einstein ring is shown with the polarisation vectors corresponding to images A and B omitted. This enables the polarisation vectors in the ring to be seen more clearly. It is interesting to see that around the majority of the ring the polarisation vectors are aligned radially outwards. As gravitational lensing preserves the orientation of polarisation on the sky (Dyer \& Shaven 1992) this is somewhat unexpected. Although it is possible that the polarisation is Faraday rotated in the jet as well as in the cores, it would be surprising if the spatial distribution of Faraday depths was such that the polarisation became aligned with the total intensity structure of the Einstein ring. However, this seems to be the case.

We have estimated the flux densities of images A and B by fitting two delta components to the $(u, v)$ data in DIFMAP Shepherd 1997). The results are shown in Table 7 . In fitting to the Stokes $I$ data we include a third component (a ring, component type 4 of the DIFMAP modelfit function) to approximate the bright Einstein ring structure and improve the fit. No such additional component was added when fitting to the Stokes $Q$ and $U$ maps as the Ein- stein ring is much fainter. Also included in Table 7 are values for the total intensities, polarised intensities and polarisation position angles as measured from the VLBI data. These are calculated from the integrated $I, Q$ and $U$ flux densities which facilitates a comparison with the values measured from the lower-resolution VLA (outer subarray) dataset. From the $P$ and $I$ measurements we calculate the corresponding percentage polarisations, $m(\%)$, which are also tabulated.

The good correspondence between the polarisation position angles as measured from the VLA and VLBI data suggests that the absolute position angle calibration has been successful. Similarly, the excellent correspondence between the integrated polarised intensities signifies that the flux scale of the two arrays are correctly aligned. However, in the VLBI maps we only detect 81 per cent of the VLA flux density in image A and 75 per cent in image B. We think this is due to a combination of the poorer surface-brightness sensitivity of the VLBI array (most of the jet visible in Fig. 1 is not detected) and over-estimation of the VLA flux densities due to blending with the ring emission in the map. This latter hypothesis is supported by the fact that we detect more of the VLA flux density in image A (which is less blended with the ring in the VLA map) as well as by the very similar polarised intensities measured by the VLA and VLBI arrays. We have made tapered maps of the VLBI data in an attempt to recover more of the A and B image flux densities. More is detected ( $\sim 5$ per cent in each image), but the VLBI flux densities of A and B still fall significantly short of their VLA values.

\section{DISCUSSION}

Our global VLBI 8.4-GHz maps have revealed a great deal of substructure in images A and B of JVAS B0218+357. Neither the lens galaxy core nor the third image were detected, both of which would be very useful constraints on the lens model, especially in this system where the centre of the lensing galaxy is currently uncertain.? In this section we discuss three main topics: the constraints that the new maps can already put on lens mass models, the evidence that the image suffers from multi-path scattering and the different polarisation distributions in each image.

\subsection{Mass model constraints}

The extensive substructure that we have detected will be useful for lens modelling and ultimately it is our intention to use the LensClean algorithm on these data for this purpose, the results of which will be presented in a future paper. In the interim we have back-projected each image into the source plane in order to qualitatively demonstrate the accuracy of the model. This has been done using the CLEAN components and an isothermal model of the lens galaxy optimized using the positions of the $15 \mathrm{GHz}$ VLBI subcomponents Patnaik et al. 1995), a flux density ratio of 3.75 and the galaxy position found from our LensClean of VLA and MERLIN data (Wucknitz2001). With $x$ and $y$ pointing along the major and minor axis of the ellipsoid, the potential is written as

$\phi=\alpha_{0} \sqrt{\frac{x^{2}}{(1+\epsilon)^{2}}+\frac{y^{2}}{(1-\epsilon)^{2}}}$.

2 The lack of detection of a third image can be used though to place an upper limit on afinite core radjus of the lens galaxy. This has been done for B0218+357 by Norbury 2002. 

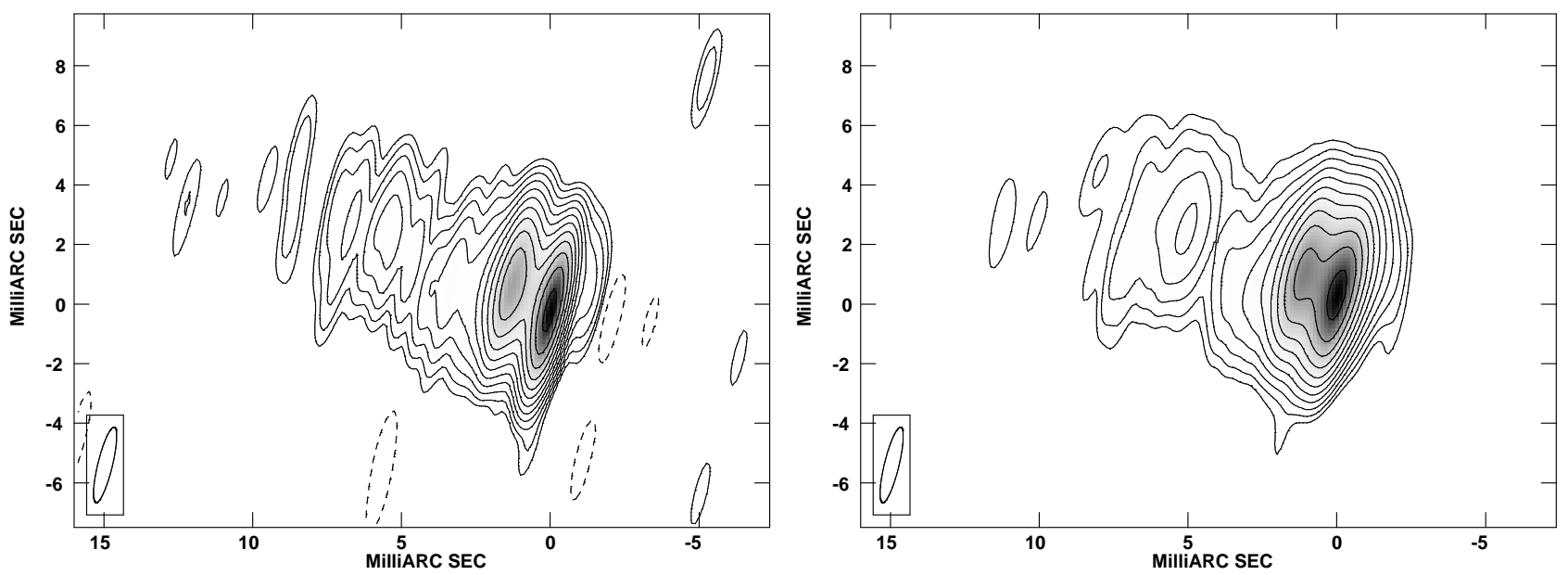

Figure 5. Maps of images A (right) and B (left) after being back-projected into the source plane. The restoring beam is shown in the bottom-left corner and has a FWHM of $2.61 \times 0.49$ mas at a position angle of -13 . 7 .

The critical radius is $\alpha_{0}=160.6$ mas, the ellipticity $\epsilon=0.0583$ and the position angle of the major axis $\theta=-41.9 \mathrm{deg}$, measured from north through east. The centre of the lens is at $z_{0}=$ $(260,117.5)$ mas relative to A.

In order to compensate for the very different effective resolution of A compared to B, the back-projected CLEAN components of both images have been restored with the same beam. This beam is just large enough that both the A and B back-projected beams are encompassed by it, which in practice means that it is approximately equal to the restoring beam in the image plane back-projected from image $\mathrm{B}$ to the source plane. This makes the nominal resolution in each the same. The back-projected maps are shown in Fig. 5 .

Compared to the lens-plane images of Fig. I the source-plane images look quite similar. Worthy of note is the excellent correspondence between the counter-components to the west of the core region which are probably images of a weak counterjet. This is intriguing though as the lensed radio source in $\mathrm{B} 0218+357$ has been classified as a BL Lac (O'Dea et al. 1992; Browne et al. 1993; Stickel \& Küh 1993), a class of objects that are not expected to display counterjets according to the unification schemes of extragalactic radio sources. This theoretical prediction is supported by the non-detection of counterjets in BL Lac sources, a notable exception being PKS 1413+135 (Perlman et al. 1994, 1996).

A closer comparison of the A and B source-plane maps shows that the $B$ jet seems to be stretched by about 10 per cent relative to the $A$ jet i.e. the jet in $B$ is longer. This stretching can only be explained with a different radial mass profile, compared to that used in the model, since the jet is directed more or less radially relative to the galaxy's centre. Writing the radial surface mass density as a function of radius as a power law, $\Sigma(r) \propto r^{-\beta}$, we find $\beta \approx$ 1.04. A very similar value is found $(\beta=1.06 \pm 0.03)$ if we use the $15-\mathrm{GHz}$ sub-component positions of Patnaik et al. 1995) as constraints Wucknitz 2001.

\subsection{Multi-path scattering}

In Fig. 5 image A looks smoother than image B. We suggest that this is due to scatter-broadening, which may also explain the frequency-dependent sizes of the images at low $(\leqslant 2.3 \mathrm{GHz})$ frequencies (Biggs et al., in preparation). Other examples of where scattering is believed to modify the surface brightness of gravitationally lensed images are PKS 1830-211 Jones et al. 1996. Guirado et al. 1999) and CLASS B1933+503 (Marlow et al.|1999). In $\mathrm{B} 0218+357$, as well as in the other two systems, the scattering would most likely originate in the lensing galaxy as we know from the observed Faraday rotation and differential depolarisation that this is both highly ionised and non-homogeneous. Also, at the lens redshift of $z=0.6847$ it is perfectly reasonable to expect that the ISM in front of each image, with a separation of 334 mas $(2.4 \mathrm{kpc})^{3}$ between them, could be significantly different.

In order to quantify the effect, we have fitted Gaussians to subcomponents 1 and 2 of the back-projected images of A and B using the task JMFIT. The deconvolved major $(a)$ and minor $(b)$ axes, as well as the position angle of the major axis $(\phi)$, are shown in Table 5 where it can be seen that for each sub-component the geometric mean of the axes (equivalent circular size) is bigger in A than in B; the area of the deconvolved A1 is approximately eight times that of B1. Also, whilst the position angles of B are as expected (they point more or less down the jet as in the image plane) those of $\mathrm{A}$ remain approximately perpendicular to this.

The advantage of model-fitting to the core sub-components in the source plane is that the back-projection removes the differences between the two images, reducing the need for removal of confusing pixels corresponding to the jet emission and rendering any bias that might exist identical in each. The disadvantage of fitting in the source plane is the potential for errors to accumulate during the many convolutions and deconvolutions that have been applied to the data at various stages of the data reduction. However, we note that the measured sizes in the image plane also show that image $\mathrm{A}$ is larger than image $\mathrm{B}$, by a factor greater than the relative magnification. From the deconvolved sizes of Table 3 the image-plane area ratios A1/B1 and A2/B2 are approximately 14 and 9. For the remainder of this analysis we only consider the source-plane size measurements.

We assume that the observed size of an A sub-component is the sum, in quadrature, of the intrinsic size and a scattering scale size $\left(\theta_{\mathrm{sc}}\right)$. We further assume that, for each sub-component, the true

3 Throughout this paper we assume a flat universe with $H_{0}=$ $70 \mathrm{~km} \mathrm{~s}^{-1} \mathrm{Mpc}^{-1}, \Omega_{0}=0.3$ and $\lambda_{0}=0.7$. 
Table 5. Deconvolved major (a) and minor (b) axes and orientation of ellipticity $(\phi)$ of Gaussians fitted to the back-projected maps as well as their geometric means and the size of the derived scattering discs $\left(\theta_{\mathrm{sc}}\right)$. All quantities are measured in mas apart from position angles which are measured in degrees. We estimate errors on the major and minor axes of about 5 per cent.

\begin{tabular}{lllrll}
\hline Image & $a$ & $b$ & $\phi$ & $\sqrt{a \times b}$ & $\theta_{\text {Sc }}$ \\
\hline A1 & 1.07 & 0.67 & 151 & 0.85 & \multirow{2}{*}{0.79} \\
B1 & 0.34 & 0.28 & 57 & 0.31 & \\
\hline A2 & 1.24 & 0.96 & 149 & 1.09 & \multirow{2}{*}{0.83} \\
B2 & 0.78 & 0.64 & 76 & 0.71 &
\end{tabular}

intrinsic size is actually that of its image B counterpart i.e. that this image is unaffected by scattering at this frequency. This assumption is supported by the similarity of the deconvolved size of B1 as measured from these data and the $15-\mathrm{GHz}$ data of Patnaik et al. (1995). The derived size of $\theta_{\mathrm{sc}}$ is also shown in Table 5 and is the same for both sub-components, 0.8 mas. The true size of the scattering disc (we have measured sub-component sizes in the source plane) is

$\hat{\theta}_{\mathrm{sc}}=\theta_{\mathrm{sc}} \frac{D_{\mathrm{s}}}{D_{\mathrm{ds}}}$

where $D_{\mathrm{s}}$ is the angular diameter distance between the Earth and the lensed source and $D_{\mathrm{ds}}$ that between the lensing galaxy and the lensed source. The relation in equation (2) is analogous to that between the true deflection angle $(\hat{\alpha})$ and the apparent deflection angle $(\alpha)$ (e.g. Refsdal \& Surdej 1994). No correction for the lens magnification is required as the displacement in the image plane due to scattering is the scattering angle magnified by the lens, and this was removed when back-projecting each image. We calculate $\hat{\theta}_{\mathrm{sc}}=3.4$ mas.

The size of the scattering disk is equal to the refractive length scale $\left(r_{\mathrm{ref}}\right)$ subtended at the distance of the lensing galaxy Narayan 1992. Using the formulation of Walker 1998) we have estimated the scattering strength (SM) of the ionised medium. With the observed frequency of $8.4 \mathrm{GHz}$ corrected to the redshift of the lensing galaxy we measure $\mathrm{SM} \approx 150 \mathrm{kpc} \mathrm{m}^{-20 / 3}$. This is a very large value compared to those seen along typical lines of sight through the Galaxy, although comparable (and in some cases much higher) values have been measured, particularly towards the Galactic centre (Cordes \& Lazio 2002). Therefore, although the scattering in front of B0218+357 image A is extreme, it is not ruled out by observations in the Galaxy.

\subsection{Depolarisation}

The polarisation properties of this system continue to intrigue. From observations of this system with MERLIN and the VLA, a general picture has emerged of image A being less polarised than image B and of both depolarising with increasing wavelength, albeit much more steeply in A. The reason for this is probably inhomogeneities in the magneto-ionic medium that is responsible for the Faraday rotation of the polarisation position angles, inhomogeneities that are greater in the region of A than B. Complications that hinder the interpretation of the polarisation results include "beating" of the polarisation position angles of sub-components 1 and 2 (for observations which do not resolve the sub-components), changing source structure with frequency and different magnification gradients across each image.

The resolution afforded by VLBI observations allows the polarisation structure of the source to be resolved and constraints to be put on the angular scale of the inhomogeneities in the Faraday screen. In Fig. 3 we see that image B, the least resolved of the images, is seen to have a higher peak polarisation ( 20 per cent) than that of A (12 per cent). This shows that we are not seeing the effects of intrinsic changes in the polarisation structures across the radio source as the lower effective resolution of B would cause these to be averaged incoherently more than in A i.e. we would expect image B to be less polarised than image A. Therefore, our global VLBI maps support the theory that the depolarisation is due to non-uniformities in the Faraday screen which are greater in front of image A. They also allow us to constrain the size of these irregularities which must be smaller than the synthesised beam i.e. $\lesssim 1$ mas. This corresponds to a linear distance of $\lesssim 7 \mathrm{pc}$ at the redshift of the lensing galaxy.

We also note that the scatter-broadening identified in Section 4.2 could cause the depolarisation. This is because scatterbroadening effectively blurs a source, causing regions with different polarisations to overlap and be averaged together. As the scattering is different in front of each image the polarisations of each will be different and, as scattering increases at longer wavelengths, the observed frequency dependence of the depolarisation could also result.

Another interpretation is that the differences between the images could result from the combination of intrinsic polarisation variability and time delay. VLA monitoring Corbett et al. 1996. Biggs et al, 1999, has shown that this source is variable in polarisation as well as in total flux density. Two-epoch VLBI observations separated by the time delay, $10.5 \pm 0.4 \mathrm{~d}$, could help to clarify the polarisation structure in this source. At the same time the picture of A being more depolarised than B is too simplistic. From earlier VLA monitoring observations Corbett et al. 1996 it was found that, with the time delay removed, the polarisation of image B was lower than that of image A at $15 \mathrm{GHz}$, the opposite to what is usually observed. This could signify time variability within the magneto-ionic medium or movement of the source relative to this.

\section{SUMMARY AND FUTURE WORK}

We have presented new maps of the lens system JVAS B0218+357 made with a global VLBI array that combine excellent sensitivity and resolution to produce the best maps yet of the mas-scale structure of this system. Unfortunately, we were unsuccessful in detecting the third image or the core of the lensing galaxy. With the new maps we have been able to explore several aspects of the lensing galaxy. From the different lengths of the jet in each image we have been able to constrain the radial mass profile and find that it is slightly steeper than isothermal $(\beta \approx 1.04)$. We also find evidence for multi-path scattering and depolarisation due to the disparity between the component sizes and polarisations in each image. These physical effects most likely result from propagation through the turbulent ionised ISM of the lensing galaxy and, in this way, B0218+357 is probably the best example of how gravitational lensing can act as a unique probe of the ISM of high-redshift galaxies.

These data will be used with LensClean (Kochanek \& Narayan 1992) in order to exploit fully the resolved structure of the images and provide further constraints for the lens model. Whilst the effects of scatter-broadening might seem to limit the usefulness of these data for lens modelling, we note that the effects of the scattering and the lens can be separated. This is because scattering only effects the sizes of components whilst the lens changes both the component sizes and separations. Therefore, future modelling will 
be able to not only constrain the lens model, but also to characterise the scattering more accurately than has been possible in this paper.

Further observations with a Global VLBI array at $5 \mathrm{GHz}$ may be worthwhile to try and detect, with higher dynamic range, the jet emission that has been revealed in the new CJ maps (Fig. 1). The present and future VLBI maps will be used to constrain the lens model, in conjunction with MERLIN, VLA and HST data. $H S T$ observations will be carried out with the Advanced Camera for Surveys which our simulations predict will give us an accuracy of $\sim 10$ mas in the galaxy position relative to the lensed images. With this vital parameter in the lens model finally measured directly (as opposed to the modelled position found from LensClean) the uncertainty in the $H_{0}$ determination from this system will be reduced to a level of about 3 per cent.

\section{ACKNOWLEDGMENTS}

The VLBA and VLA are operated by the National Radio Astronomy Observatory which is a facility of the National Science Foundation operated under cooperative agreement by Associated Universities, Inc. The European VLBI Network is a joint facility of European and Chinese radio astronomy institutes funded by their national research councils. We would like to thank the staff of the DSN at Goldstone and Robledo for their assistance in making these observations possible. This research was supported in part by the European Commission TMR Programme, Research Network Contract ERBFMRXCT96-0034 'CERES'. OW was partially funded during this work by the European Commission, Marie Curie Training Site programme, under contract no. HPMT-CT-2000-00069. ADB would like to thank the staff of JIVE for their help with writing the observing schedule. Much thanks goes to Alok Patnaik for his contribution to the proposal as well as for his help with the initial stages of the data reduction. We also thank the anonymous referee for a number of suggestions and comments that improved the paper.

\section{REFERENCES}

Biggs A.D., Browne I.W.A., Helbig P., Koopmans L.V.E., Wilkinson P.N., Perley R.A., 1999, MNRAS, 304, 349

Biggs A.D., Browne I.W.A., Muxlow T.W.B., Wilkinson P.N., 2001, MNRAS, 322, 821

Browne I.W.A., Patnaik A.R., Walsh D., Wilkinson P.N., 1993, MNRAS, 263, L32

Chen G.H., Hewitt J.N., 1993, AJ, 106, 1719

Chen G.H., Kochanek C.S., Hewitt J.N., 1995, ApJ, 447, 62

Cohen A.S., Hewitt J.N., Moore C.B., Haarsma D.B., 2000, ApJ, 545,578

Corbett E.A., Browne I.W.A., Wilkinson P.N., Patnaik A.R., 1996, in Kochanek C.S., Hewitt J.N., eds., Proc. IAU Symp. 173, Astrophysical Applications of Gravitational Lensing. Kluwer, Dordrecht, p. 37

Cordes J.M., Lazio T.J.W., 2002, astro-ph/0207156

Courbin F., Saha P., Schechter P., 2002, astro-ph/0208043

Dyer C.C., Shaver E.G., 1992, ApJ, 390, L5

Fassnacht C.D., et al., 1999, AJ, 117, 658

Freedman W.L., et al., 2001, ApJ, 553, 47

Guirado J.C., Jones D.L., Lara L., Marcaide J.M., Preston R.A., Rao A.P., Sherwood W.A., 1999, A\&A, 346, 392
Harvanek M., Stocke J.T., Morse J.A., Rhee G., 1997, AJ, 114, 2240

Ibata R.A., Lewis G.F., Irwin M.J., Lehár J., Totten E.J., 1999, AJ, 118,1922

Jones D.L., et al., 1996, ApJ, 470, L23

Kemball A.J., Patnaik A.R., Porcas R.W., 2001, ApJ, 562, 649

Kochanek C.S., Narayan R., 1992, ApJ, 401, 461

Lehár J., et al., 2000, ApJ, 536, 584

Lewis G.F., Ibata R.A., Ellison S.L., Aracil B., Petitjean P., Pettini M., Srianand R., 2002, MNRAS, 334, L7

Marlow D.R., Browne I.W.A., Jackson N., Wilkinson P.N., 1999, MNRAS, 305, 15

Narayan R., 1992, Phil. Trans. R. Soc. Lond., 341, 151

Norbury M., 2002, Ph.D. thesis, Victoria University of Manchester

O’Dea C.P., Baum S.A., Stanghellini C., Dey A., van Breugel W., Deustua S., Smith E.P., 1992, AJ, 104, 1320

Parodi B.R., Saha A., Sandage A., Tammann G.A., 2000, ApJ, 540, 634

Patnaik A.R., Browne I.W.A., King L.J., Muxlow T.W.B., Walsh D., Wilkinson P.N., 1993, MNRAS, 261, 435

Patnaik A.R., Porcas R.W., Browne I.W.A., 1995, MNRAS, 274, L5

Perlman E.S., Stocke J.T., Shaffer D.B., Carilli C.L., Ma C., 1994, ApJ, 424, L69

Perlman E.S., Carilli C.L., Stocke J.T., Conway J., 1996, AJ, 111, 1839

Porcas R.W., Patnaik A.R., 1996a, in Kochanek C.S., Hewitt J.N., eds., Proc. IAU Symp. 173, Astrophysical Applications of Gravitational Lensing. Kluwer, Dordrecht, p. 311

Porcas R.W., Patnaik A.R., 1996b, in Ekers R.D., Fanti C., Padrielli L., eds., Proc. IAU Symp. 175, Extragalactic Radio Sources. Kluwer, Dordrecht, p. 115

Refsdal S., 1964, MNRAS, 128, 307

Refsdal S., Surdej J., 1994, Rep. Prog. Phys., 57, 117

Saikia D.J., Salter C.J., 1988, ARA\&A, 26, 93

Schechter P.L., 2000, in Lasenby A.N., Jones A.W., Wilkinson A., eds., Proc. IAU Symp. 201, New Cosmological Data and the Values of the Fundamental Parameters. Astron. Soc. Pac., San Francisco, in press

Shanks T., Allen P.D., Hoyle F., Tanvir N.R., 2001, in Shanks T., Metcalfe N., eds., ASP Conf. Ser., A New Era in Cosmology. Astron. Soc. Pac., San Francisco, in press

Shepherd M.C., 1997, in Hunt G., Payne H.E., eds., ASP Conf. Ser. Vol. 125, Astronomical Data Analysis Software and Systems VI. Astron. Soc. Pac., San Francisco, p. 77

Stickel M., Kühr H., 1993, A\&AS, 101, 521

Walker M.A., 1998, MNRAS, 294, 307

Winn J.N., et al., 2002, AJ, 123, 10

Wucknitz O., 2001, in Shanks T., Metcalfe N., eds., ASP Conf. Ser., A New Era in Cosmology. Astron. Soc. Pac., San Francisco, in press

Xu W., Readhead A.C.S., Pearson T.J., Polatidis A.G., Wilkinson P.N., 1995, ApJS, 99, 297 